\title{
Réflexion sur les modifications du maillage communal francais depuis 1790
}

Modifications to the french communal network since 1790

Überlegung zu den Veränderungen des Gemeindenetzes seit 1790 in Frankreich

\section{Simon Edelblutte}

\section{(2) OpenEdition}

Journals

Édition électronique

URL : http://journals.openedition.org/rge/4039

DOI : $10.4000 /$ rge.4039

ISSN : $2108-6478$

Éditeur

Association des géographes de l'Est

Édition imprimée

Date de publication : 1 septembre 2000

ISSN : 0035-3213

\section{Référence électronique}

Simon Edelblutte, "Réflexion sur les modifications du maillage communal francais depuis 1790 », Revue Géographique de l'Est [En ligne], vol. 40 / 4 | 2000, mis en ligne le 27 juillet 2013, consulté le 08 septembre 2020. URL : http://journals.openedition.org/rge/4039 ; DOI : https://doi.org/10.4000/rge 4039

Ce document a été généré automatiquement le 8 septembre 2020

Tous droits réservés 


\title{
Réflexion sur les modifications du maillage communal francais depuis
} 1790

\author{
Modifications to the french communal network since 1790 \\ Überlegung zu den Veränderungen des Gemeindenetzes seit 1790 in Frankreich
}

\section{Simon Edelblutte}

1 Alors que de nombreux travaux s'attachent au récent et fort développement des structures intercommunales, les modifications des limites communales sont souvent négligées car la commune, maille administrative de base de l'espace français, est aujourd'hui considérée comme un cadre obsolète. Le maillage communal mis en place en 1790 est en effet inadapté à l'organisation spatiale actuelle et «il n'y a plus guère de fondement économique à cet univers des communes françaises»(Di Méo, 1993). Résistant le plus souvent aux nombreuses tentatives de réformes visant à le faire évoluer brusquement, il s'est néanmoins ponctuellement adapté à des situations locales particulières, notamment lorsque la maille communale correspondait très mal ou ne correspondait plus du tout à l'organisation spatiale.

2 Ces changements de limites communales, mineurs lorsqu'il s'agit d'un simple déplacement de la limite ou majeurs quand une commune disparait ou apparaît, sont peu connus et considérés comme peu significatifs par rapport aux nombreuses formes et tentatives d'intercommunalité aujourd'hui mises en exergue. Pourtant, ces modifications, en réalité assez nombreuses ${ }^{1}$, sont riches d'enseignements. Ne sont-elles pas en effet autant d'adaptations d'un cadre administratif réputé figé à l'évolution permanente de l'organisation spatiale, et surtout à la naissance et au déclin de géosystèmes successifs, à leur « dérive"? (Humbert, 1994). Terme fréquemment utilisé en géographie physique pour désigner des systèmes produits par l'action conjuguée du relief, du climat et des sols, le géosystème est donc un espace cohérent aux éléments constitutifs interdépendants et aux liens étroits, s'inscrivant dans le paysage. A ce titre, il peut être utilisé en géographie humaine pour désigner des ensembles homogènes et cohérents lisibles dans le paysage, tels que les finages d'avant 
le déclin rural (Humbert, 1994), mais aussi les ensembles industriels nés autour des usines au XIXe siècle, formant un géosystème d'un nouveau genre (Edelblutte, 1998), ou peut-être encore d'autres ensembles plus vastes, construits autour des agglomérations urbaines actuelles (Edelblutte, 1997).

Une recomposition politique du territoire peut donc parfois suivre une recomposition, économique, démographique, sociale... de ce même territoire. L'objectif de cet éclairage historique des modifications de la maille administrative locale est donc de tenter d'expliquer les adaptations ponctuelles de cette maille à la recomposition permanente des territoires; d'abord en présentant cette idée de l'adaptation de la maille communale à l'organisation spatiale et au géosystème et en évoquant ses limites, car ces adaptations sont loin d'être la règle générale ; ensuite, en développant, avec l'appui d'exemples choisis dans toute la France ${ }^{2}$, les trois principales causes des modifications, c'est-à-dire le développement de l'industrialisation, la poussée urbaine et les flux et reflux du peuplement rural. Ce travail propose donc un cadre théorique liant les modifications des limites communales aux changements de l'organisation spatiale. Cependant, cette première approche, nécessairement succincte dans le cadre d'un article, devra être étayée, développée et débattue ultérieurement par l'étude d'autres exemples.

\section{La recomposition du territoire administratif local : une adaptation ponctuelle et incomplète aux changements de l'organisation spatiale}

4 L'organisation spatiale d'un lieu évolue en permanence, «les territoires étant une manifestation possible, au mieux une composante momentanément stable de la dynamique des systèmes en interaction" (Reymond, 1996); cette évolution subit parfois des accélérations qui provoquent des tensions croissantes entre les réalités économiques, démographiques, voire sociales ou culturelles, et le cadre administratif, territoire politique. Cette inadaptation croissante se traduit parfois par une recomposition de la maille communale. Ces modifications sont importantes, car un territoire dont le cadre administratif est mal adapté à la réalité de l'organisation spatiale fonctionne mal et subit des contraintes qui peuvent freiner son développement ${ }^{3}$.

\section{A. 1790 : la commune correspond à un géosystème, mais des ajustements sont déjà nécessaires}

5 A la Révolution, les quelques 44000 communes créées correspondent aux géosystèmes existants. En effet, elles ne sont que le prolongement direct des finages établis sur les collectes fiscales et/ou sur les paroisses ${ }^{4}$ de l'Ancien Régime, plongeant elles-mêmes leurs racines bien au-delà (Di Méo, 1991; Mathis, 2001). Certes, leur statut diffère totalement de celui des communautés pré-révolutionnaires, mais leur territoire est le même. La commune se calque donc sur le finage, géosystème agricole bien inscrit dans le paysage, à l'enveloppe (les limites) précise, au cœur (le chef-lieu) évident, à la tête (l'église) bien identifiée, aux organes constitutifs (les différents terroirs complémentaires, la forêt, les jardins) interdépendants et aux liens avec l'extérieur (les 
routes et chemins) encore ténus. Il existe ainsi une continuité entre le maillage de base de l'Ancien Régime et celui du nouveau, contrairement aux échelons administratifs supérieurs où la rupture est beaucoup plus forte entre provinces et départements. Fondé sur une économie agricole d'autoconsommation, le tissu communal initial est donc en phase avec l'organisation socio-économique de l'époque.

Cependant, les tensions, sources de modifications de la maille communale, existent dès la Révolution, lorsque les communes ne correspondent pas ou correspondent mal aux géosystèmes agricoles ou aux quelques géosystèmes urbains. C'est le cas lorsqu'une ville et la campagne environnante formaient une seule communauté sous l'Ancien Régime. Après 1790 , deux géosystèmes cohabitent donc sur une même commune, ce qui peut entraîner son démembrement rapide. Ainsi furent créées, rien qu'en Lozère, département pourtant peu urbanisé, mais aux territoires communaux assez étendus, trois communes « foraines », en opposition aux communes « ville » : le Malzieu-Forain, Meyrueis-Forain et Saint-Chély-Forain. D'autres ajustements concernent, entre 1790 et 1815, des " communes floues ", c'est-à-dire des communes créées, disparues, recréées, à plusieurs reprises avant 1815. Ainsi, dans la Meuse 4 communes sont créées en $1794 . .$. pour finalement disparaître en 1810 ! Cette instabilité peut être liée à leur population un peu trop forte pour n'être qu'une simple partie d'un géosystème, mais insuffisante pour constituer autour de chacune d'elles un véritable géosystème. Enfin, d'autres adaptations existent, dans des départements à l'habitat dispersé et au relief contraignant, composés de vastes communes aux écarts très éloignés du chef-lieu et d'accès difficile. Dans ce cas, ces écarts qui n'étaient pourtant pas des communautés distinctes sous l'Ancien Régime et qui ne correspondent pas à la définition du géosystème, ont pu être érigées en communes. Dans ce cas, l'éloignement et la coupure administrative ont pu, si la nouvelle commune finit par regrouper un nombre suffisant d'habitants, générer un nouveau géosystème, inversant la théorie exposée en introduction et méritant débat.

7 Ces premiers ajustements, assez fréquents entre 1790 et 1815, montrent que la trame communale, généralement bien adaptée aux nombreux géosystèmes agricoles et aux quelques géosystèmes urbains, était cependant parfois modifiée en fonction des recompositions territoriales en cours.

\section{$B$ De nombreux freins génèrent une inadaptation croissante de la trame communale à l'organisation spatiale}

8 Les quelques 44000 communes de 1790 ne sont plus en l'an 2000 que $36566^{5}$. La tendance dominante est donc à la réduction du nombre total de communes, suivant en cela, mais avec beaucoup d'hésitations et de retard, la réduction du nombre de géosystèmes, elle-même liée à l'extension des organismes urbains et au déclin puis à la disparition des ensembles agricoles hérités de l'Ancien Régime; la naissance de nouveaux géosystèmes, industriels, touristiques ou urbains, n'ayant pu compenser, en quantité, l'effacement des géosystèmes agricoles.

9 Cependant, et malgré ces ajustements, la trame communale est de plus en plus inadaptée à l'organisation spatiale. En effet, la disparition progressive des géosystèmes agricoles et la naissance et le développement de géosystèmes urbains et industriels n'ont pas toujours généré des modifications communales, ou ont généré des modifications incomplètes, imparfaites. Tous les changements de limites 
communales seraient donc bien des tentatives d'adaptations de la maille administrative de base aux changements de l'organisation spatiale, mais tout changement de l'organisation spatiale ne génère pas de modification de la maille communale. En effet, il existe donc de nombreux obstacles à l'adaptation du tissu communal à l'organisation spatiale. Le poids de la tradition, de la coutume, du vécu des habitants et donc de l'Histoire, est à mettre au premier rang de ces freins. En effet, " plus l'histoire d'un système est profonde, plus il résiste aux pressions transformantes qu'il subit " (Humbert, 1994). Les limites communales nées sur ces systèmes très anciens sont donc, elles aussi, très résistantes.

L'État a également joué un rôle majeur dans l'adaptation ou l'inadaptation de la trame communale à l'organisation spatiale, qu'il a parfois favorisé, parfois contrecarré, avec le plus souvent peu de succès. A deux reprises, les gouvernements ont appuyé la tendance lourde à la réduction du nombre de communes, suivant en cela la réduction du nombre de géosystèmes ; d'abord, entre 1810 et $1848^{6}$, pour des raisons financières, mais aussi pratiques, car de nombreuses petites communes ne comptaient aucun citoyen sachant lire ou écrire ; ensuite, dans les années 1960 et 1970, en réponse à l'anémie démographique de nombreuses communes rurales, avec une première loi en 1959, puis surtout avec la loi Marcellin de 1971, comportant de nombreuses mesures incitant les communes à la fusion. Durant ces deux périodes, les fusions ont été effectivement plus nombreuses, mais n'ont jamais atteint le nombre espéré et ont souvent débouché, à cause des freins évoqués ci-dessus, sur des échecs. Des exemples seront développés dans les parties suivantes. Inversement, l'État, notamment entre 1848 et 1958, a favorisé le maintien, voire la création de communes, souvent en contradiction avec l'évolution de l'organisation spatiale. Avec le suffrage universel, progressivement appliqué dans la deuxième moitié du XIXe siècle, l'État a protégé «l'émiettement favorisant le clientélisme politique cher aux députés de province » (Poitou, 2000), conservant une trame communale de plus en plus inadaptée à l'organisation spatiale, caractérisée par le déclin très fort des géosystèmes agricoles.

11 Malgré ces freins et à cause ou en dépit des incitations contradictoires de l'État, le maillage communal, né sur une organisation spatiale liée à l'agriculture, a donc connu diverses séries d'adaptations majeures ou mineures, essentiellement liées à l'industrialisation, à l'urbanisation, et plus récemment au dépeuplement des campagnes.

\section{Le rôle majeur de l'industrialisation}

Dans les différentes causes de modification de limites communales, le développement industriel, à partir du milieu du XIXe siècle, joue un rôle prépondérant. En effet, avec le développement de toute une organisation spatiale fondée sur l'industrie, comprenant un cœur (l'usine), une tête (la maison du patron), des organes divers (les cités ouvrières, les bâtiments sociaux), des liens avec l'extérieur (les routes, les voies ferrées, les canaux), c'est un géosystème industriel qui se développe, c'est-à-dire un organisme cohérent, bien délimité et bien inscrit dans le paysage. Les limites du nouveau géosystème sont évidemment différentes de celles de son ou ses prédécesseurs agricoles et deux types de recomposition du territoire administratif peuvent alors intervenir, allant de la modification des limites communales déjà existantes à la création d'une nouvelle commune. 


\section{A. Les modifications de limites : le géosystème industriel supplante le géosystème agricole}

13 Face à l'affirmation du géosystème industriel, la maille communale fondée sur le géosystème agricole vidé de sa substance, ne convient plus. Des tensions apparaissent, générant des modifications des limites communales. Parfois simple échange de territoire ${ }^{7}$, ces changements sont souvent de véritables annexions de vastes territoires de communes voisines restées plus rurales, par la commune ayant accueilli l'usine, cœur du nouveau géosystème. Ces adaptations sont facilitées par la présence très fréquente, au poste de maire, du propriétaire de l'usine qui tient donc à ce que toutes les composantes du géosystème industriel dont il est l'âme et le dirigeant, se trouvent sur un seul et même territoire.

La figure 1 présente le cas de Neuves-Maisons, en Meurthe-et-Moselle, à quelques kilomètres au sud de Nancy. Petite commune viticole, Neuves-Maisons, accueille en 1872 une grosse usine sidérurgique, installée à proximité de la Moselle. Si les premières cités ouvrières trouvent de la place sur la commune même, les suivantes, édifiées en 1895, débordent largement sur la commune voisine de Chaligny. Arguant de leur éloignement du village de Chaligny et de leur proximité de l'usine, le maire - et patron de l'usine -, réussit à faire détacher en 1901 de Chaligny une soixantaine d'hectares. En 1910, la même opération se répète aux dépens d'une autre commune voisine, Chavigny, qui perd 75 ha sur lesquels se trouvent des cités ouvrières et surtout la mine du Val-deFer approvisionnant l'usine néodomienne en minerai. Le territoire communal s'est donc adapté à la croissance et à la puissance grandissante du géosystème industriel né autour de l'usine puisque l'essentiel de ses composantes, de la mine à l'usine en passant par la grande majorité des cités, les bâtiments économiques et sociaux (économats, coopératives, infirmerie de l'usine...) ont fini par se trouver sur le même territoire communal, facilitant le fonctionnement du géosystème industriel à son apogée. Cet exemple montre enfin l'importance de la présence d'un leader, homme d'influence poussant les autorités à accepter la révision de la trame communale. 
Figure 1 : L'adaptation des limites communales au géosystème néodomien au début du XXe siècle

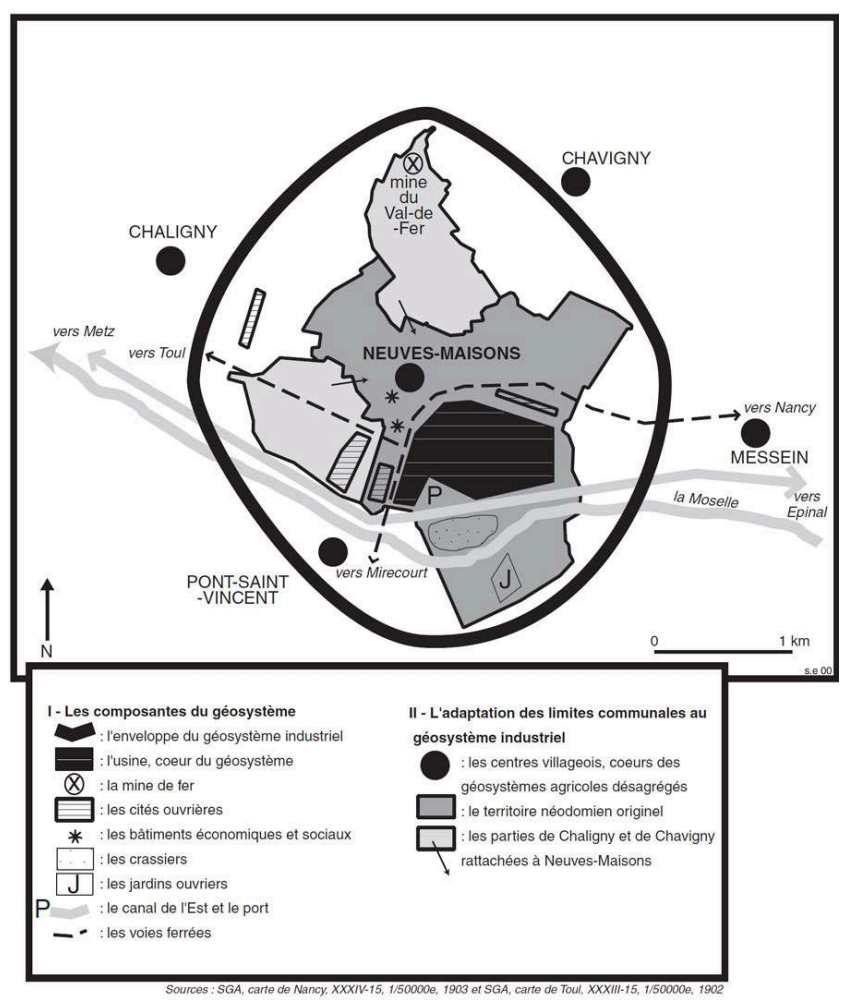

\section{B. La création de nouvelles communes : deux géosystèmes sur une même commune}

La présence, sur un même territoire communal, de deux géosystèmes différents génère de vives tensions et se traduit souvent par l'érection en commune de la partie industrielle qui ne peut plus s'entendre avec la partie agricole. A l'échelle communale, la réussite de cette recomposition majeure du territoire est favorisée par une superficie importante de la commune initiale et surtout par la dispersion de l'habitat au sein de cette commune. L'usine peut en effet s'installer près d'un hameau (voire de façon totalement isolée), loin du chef-lieu de la commune. La dualité de l'organisation spatiale joue alors à plein et il ne manque plus que des limites communales nouvelles pour entériner ce nouvel organisme détaché de l'ancien géosystème.

16 La figure 2 développe l'exemple de la commune du Pont-de-Claix, dans l'Isère, née en 1873 à partir des communes de Claix et de Champagnier, à quelques kilomètres au sud de Grenoble. Une usine chimique s'installe aux confins des deux communes, toutes deux assez étendues, près d'un écart qui s'était développé sur un point de franchissement du Drac. L'usine est non seulement «à l'origine de l'histoire et du paysage urbain » (Donze, 1996) du Pont-de-Claix, mais bien aussi de la commune ellemême, créée sous la pression de l'industriel. La grande taille de la commune, l'éloignement du site industriel par rapport aux chefs-lieux villageois, mais aussi la volonté des industriels d'exercer une domination non seulement économique, mais aussi administrative, sur le géosystème qu'ils ont créé, sont donc à l'origine du Pont-deClaix, dont le territoire est aujourd'hui inclus dans l'agglomération grenobloise. 
Figure 2 : Le Pont-de-Claix à la fin du XIXe siècle : du nouveau géosystème à la naissance d'une nouvelle commune

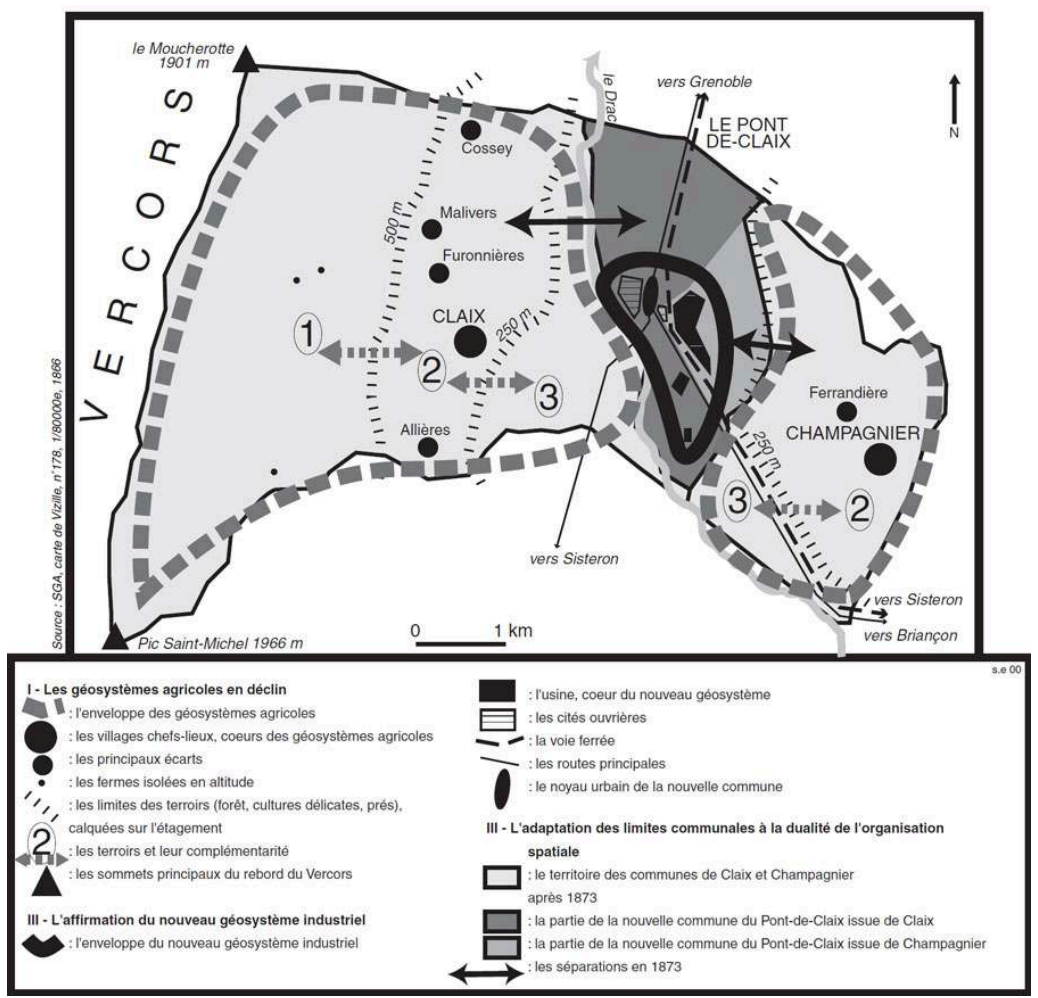

\section{Des adaptations manquées}

Les exemples de créations de communes et de modifications de limites communales pourraient être multipliés ${ }^{8}$, tendant à renforcer le postulat de départ, c'est-à-dire la nécessaire adaptation des limites communales à de nouveaux géosystèmes ou à l'évolution des anciens. Cependant, il existe également de nombreux cas où l'adaptation ne se produit pas ou se produit incomplètement ou encore imparfaitement. Le plus fréquent est l'existence avérée d'un géosystème industriel ne générant pas la naissance d'une nouvelle commune (Edelblutte, 2000), pour des raisons variées, dont la principale est certainement l'absence d'un leader conduisant les séparatistes.

Un cas plus rare, mais très révélateur des liens entre l'organisation spatiale et les limites communales, illustre la difficulté de faire vivre une nouvelle commune si elle ne s'adapte pas bien à un géosystème existant. Cité ouvrière construite de toutes pièces entre 1952 et 1957 sur les territoires de Ranguevaux et de Fameck par l'entreprise sidérurgique SOLLAC, Saint-Nicolas-en-Forêt (Moselle) est liée aux deux usines de Sérémange-Erzange et de Florange, où la SOLLAC emploie alors plus de 10000 personnes (Reuter, 1972). La création de la nouvelle commune en 1958 est justifiée à l'origine par le caractère entièrement nouveau de cette urbanisation et par l'éloignement des chefs-lieux des communes de rattachement; elle est également une des dernières manifestations du paternalisme des industriels lorrains. Cette commune ne s'étend en réalité que sur une partie restreinte d'un vaste géosystème industriel largement intercommunal. En effet, les usines situées à Florange et Sérémange-Erzange recrutent très au-delà des limites de ces deux communes et ne sont que la partie aval du géosystème minier et sidérurgique présenté sur la figure 3 . Dès sa création, le 
territoire nicoforestier ne correspond donc absolument pas aux limites du géosystème dominant à l'époque; de plus cette création récente n'a pas, contrairement aux communes voisines antérieures à l'industrialisation - et donc elles-mêmes inadaptées à l'organisation spatiale dominante -, de fondements historiques reconnus pour préserver son existence. Sans ressources importantes pour équiper son territoire et satisfaire une population voisine de 3500 habitants (1968), la commune demande son rattachement à la ville voisine d'Hayange, ce qui lui est accordé en 1970.

Figure 3 : Saint-Nicolas-en-Forêt (1958-1970) : l'échec d'une création communale sur une partie d'un vaste géosystème

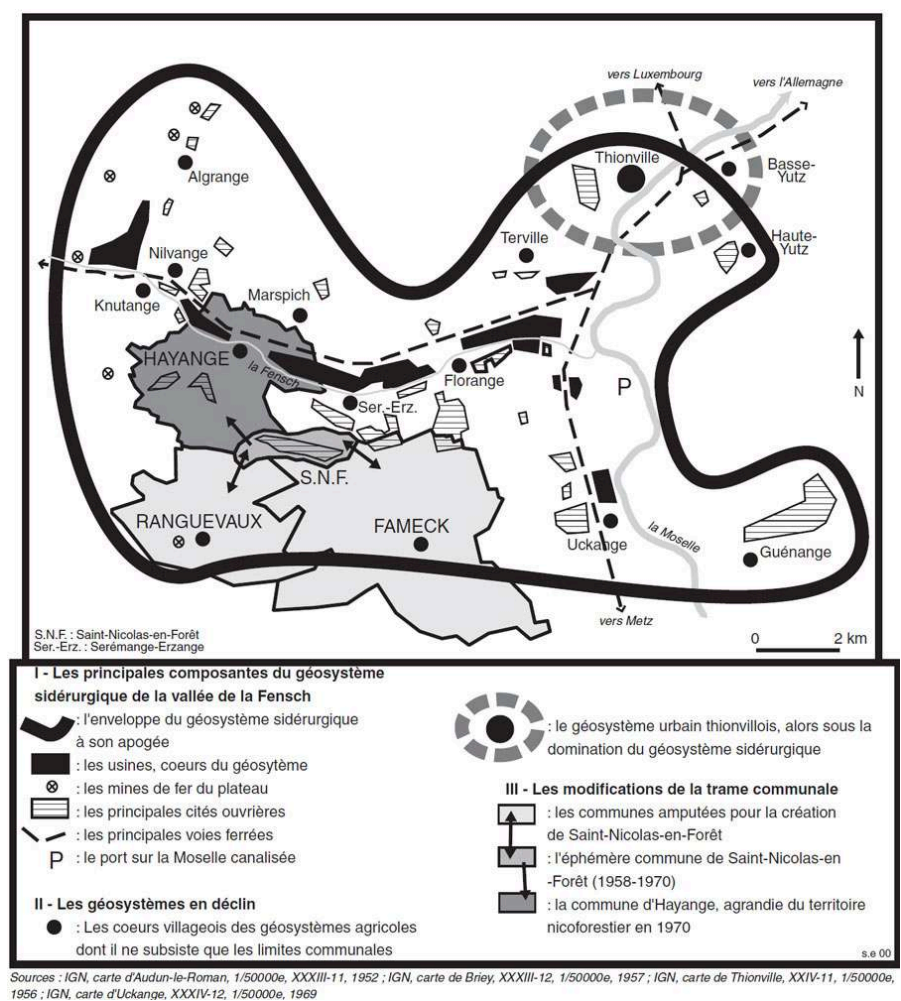

Ce dernier cas est donc très révélateur de la difficulté de faire vivre une nouvelle commune sans qu'il y ait correspondance entre son territoire et un nouveau géosystème et sans le poids de l'Histoire pour justifier son existence. Le même problème se retrouve autour des villes, autres lieux de profondes modifications communales depuis la fin du XIXe siècle.

\section{Le poids croissant de l'urbanisation}

Tout comme l'industrie, la poussée de l'urbanisation, très sensible à partir de la fin du XIXe siècle, remet en cause les limites héritées de l'Ancien Régime et fixées dans la maille communale à la Révolution. Le même processus d'adaptation des limites aux nouveaux géosystèmes urbains en croissance est à l'œuvre. Il s'effectue de deux façons souvent successives, parfois concomitantes ; d'abord par des créations de communes en périphérie, puis par l'agrandissement de la commune-centre. 


\section{A. Les modifications dans la banlieue, résultats de la dislocation des géosystèmes agricoles}

Les communes urbaines nées de la Révolution ont calqué leurs limites sur les géosystèmes urbains préexistants, incluant, au-delà des fortifications, quelques espaces agricoles non bâtis, dépendant de la ville et la ravitaillant. La croissance urbaine du XIXe siècle remet en cause cette organisation en s'étalant d'abord sur les parties agricoles de la commune, puis, surtout, en débordant sur le territoire des communes rurales voisines.

La banlieue parisienne, embryonnaire à la Révolution, s'étend surtout au XIXe siècle, provoquant une multitude de modifications mineures de limites communales ${ }^{9}$, et surtout la création de nouvelles communes tant en banlieue proche qu'en banlieue plus lointaine. La figure 4 place toutes les communes créées depuis 1815, fin de la période d'instabilité initiale des limites, dans les départements actuels de la petite couronne. Le mouvement s'amorce en 1830 aux portes de la commune capitale par la naissance de Batignolles-Monceaux et de Grenelle, et se poursuit avec 26 créations, la dernière intervenant, dans la grande couronne, en 1977, avec la naissance de la commune des Ulis (Essonne). Ces nouvelles communes ne correspondent en réalité pas à de nouveaux géosystèmes, mais plutôt à l'empiétement du géosystème parisien sur les anciens géosystèmes agricoles qui se désagrègent. Le mouvement de création a été très marqué à la charnière des XIXe et XXe siècles, correspondant à la fois à une forte croissance urbaine et à la faveur des autorités pour ces naissances communales. Cependant, même lorsque que les gouvernements favorisent les fusions, l'exceptionnelle croissance urbaine parisienne entraîne des créations de communes, comme celle des Ulis évoquée ci-dessus. Le même phénomène se produit dans de nombreuses villes de province, aux dépens des communes de banlieue, mais aussi et parfois aux dépens d'une communecentre au territoire plus étendu. Ainsi, Morières et le Pontet se détachent-elles d'Avignon en 1870 et 1925 (fig. 5). Dans ce dernier cas, la séparation est favorisée par le transfert d'usines textiles et de main d'œuvre d'Avignon vers le Pontet et n'est réussie que grâce à la présence d'une forte personnalité à la tête du mouvement séparatiste, $\mathrm{M}$. Thomas, "riche héritier oisif d'une lignée industrielle avignonnaise» (Dréano, 1996-98). Il s'est donc constitué ici un géosystème industrialo-urbain, organisé différemment du géosystème avignonnais, mais dont le développement est tout de même très dépendant de la croissance urbaine avignonnaise. 
Figure 4 : Les modifications majeures des limites communales dans la petite couronne parisienne

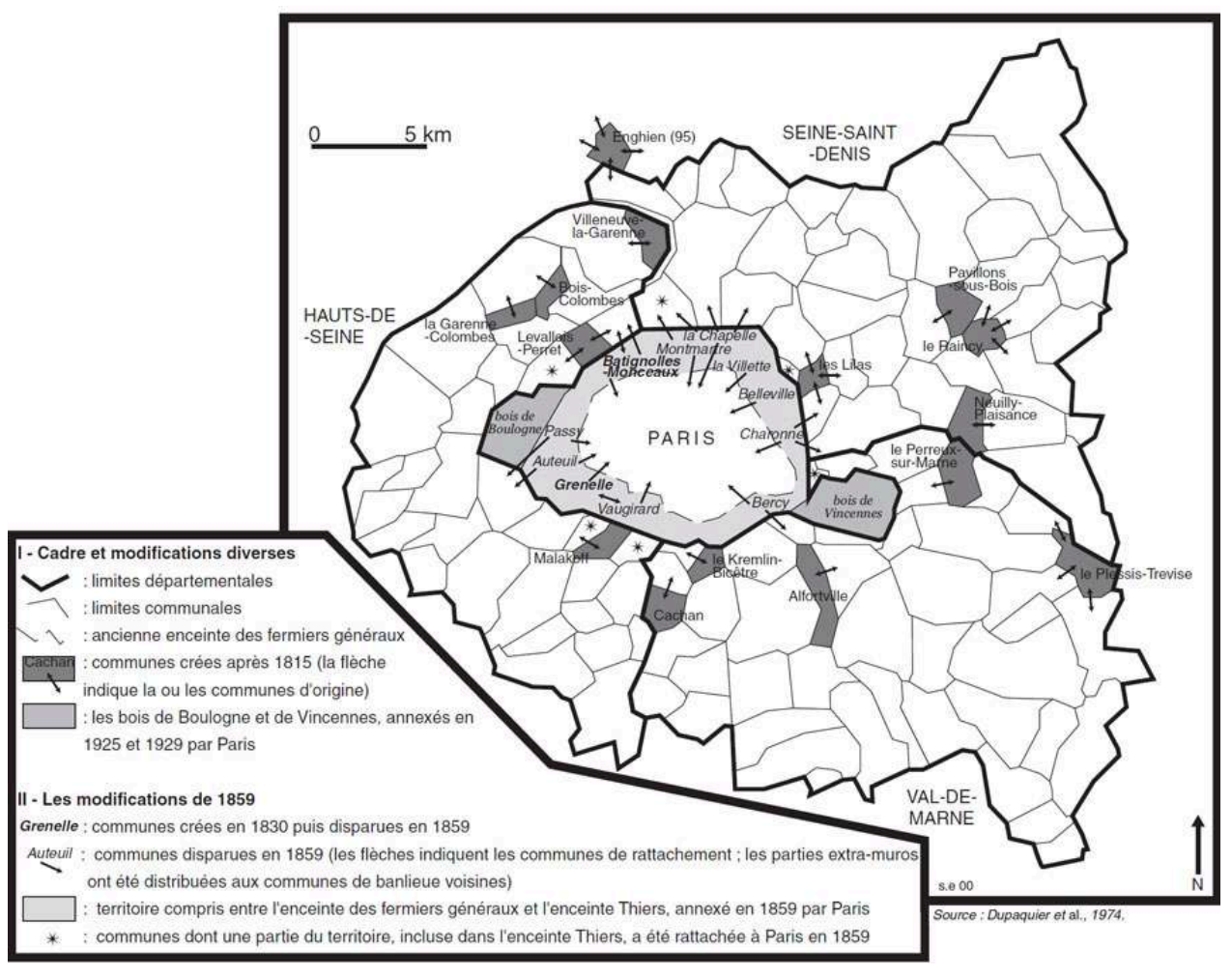

Figure 5 : La recomposition du maillage communal en Avignon (fin XIXe-début XXe)

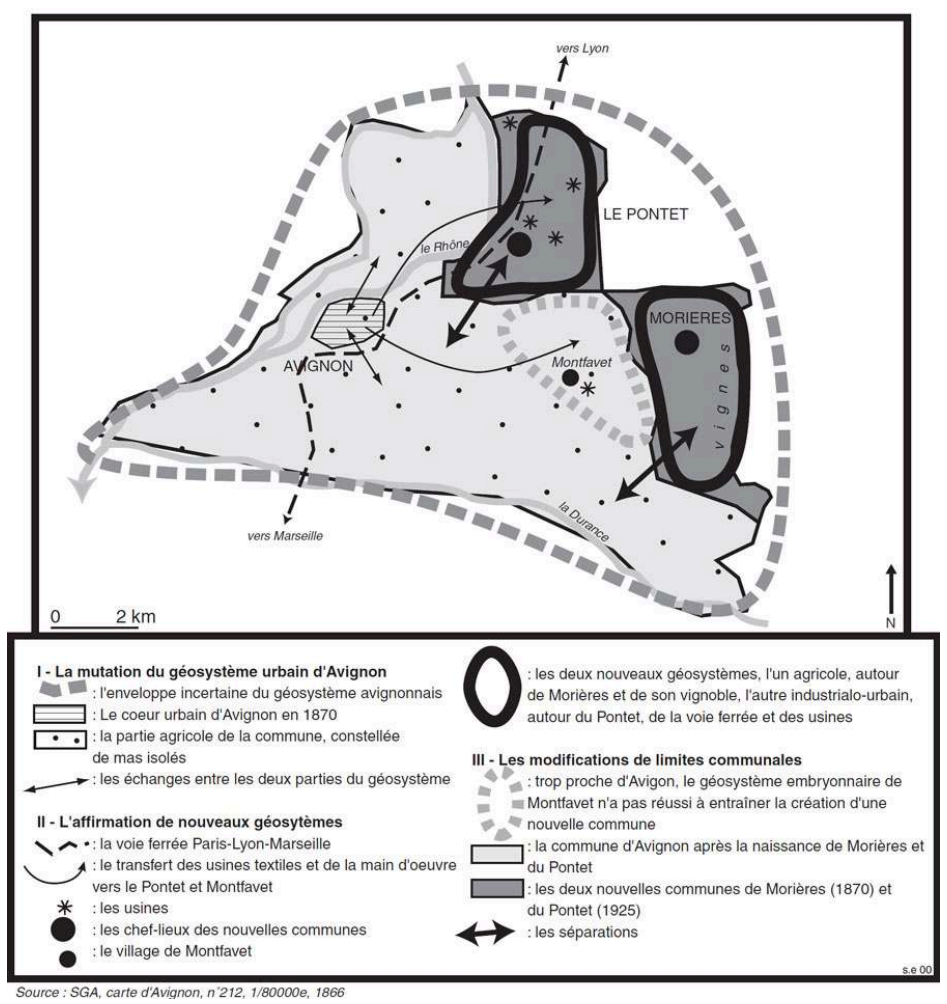


23 Par la suite, et alors que se renforce le géosystème urbain dominé par la ville principale, les créations de communes se font plus rares, alors que se multiplient les disparitions de communes de banlieues, absorbées par la commune-centre.

\section{B. L'agrandissement de la commune centre : l'adaptation au géosystème urbain}

24 Les géosystèmes urbains actuels sont bien plus vastes que les géosystèmes agricoles ou les géosystèmes industriels de la fin du siècle dernier. La ville étend en effet son influence visible sur des territoires éloignés de plusieurs dizaines de kilomètres, comme par exemple les villages rurbanisés. Les limites de ces nouveaux géosystèmes sont donc assez floues et le maillage communal peine souvent à s'y adapter. Cependant, les exemples d'absorption de communes de banlieue par les communes-centres sont en réalité très nombreux, et encore une fois, l'exemple parisien apparaît comme pionnier, bien qu'assez atypique par le blocage des modifications après 1860 .

Entouré de l'enceinte des fermiers généraux construite sous Louis XVI, la commune de Paris ne correspondait déjà plus, à la Révolution, au géosystème urbain plus étendu dont elle était le centre. Une nouvelle enceinte, correspondant au périphérique actuel, est construite par Thiers sous la Monarchie de Juillet, incluant les premiers quartiers suburbains parisiens. En 1859, soit plus de 10 ans après la construction de l'enceinte, la trame communale est adaptée à la nouvelle réalité géographique par la disparition des 11 communes entièrement ou presque entièrement incluses dans cette nouvelle enceinte et l'annexion de parties de six communes également incluses dans cette enceinte (fig. 4). Le cas des communes de Batignolles-Monceaux et Grenelle, créées hors enceinte en 1830 à partir de Clichy et de Vaugirard - et donc issues de la poussée urbaine parisienne hors les murs - puis absorbées par Paris en 1859, illustre bien l'adaptation en deux étapes - création de nouvelles communes en banlieue, puis absorption des communes de banlieue par la commune-centre - de la maille communale à la croissance des géosystèmes urbains.

En province, la croissance des territoires communaux urbains est plus tardive et parfois même assez récente, profitant de la faveur pour les fusions constatée après 1960 . De nombreuses villes, de toutes tailles, ont annexé, soit des parties de communes voisines, comme Boulogne-sur-Mer, soit des communes entières. Dunkerque et Thionville en absorbent ainsi quatre, Saint-Etiennne et Saumur, trois, Tours et Béthune, deux, et Metz, six, en deux phases. La première est liée à l'occupation allemande qui a favorisé la croissance de la commune messine en lui annexant Plantières et Devant-les-Ponts en 1907 et Le Sablon en 1914; la seconde, plus habituelle pour une ville française, intervient en 1961 avec l'absorption de Vallières-lès-Metz, Borny et Magny.

Cet agrandissement de la commune urbaine centrale dont les limites tendent à s'adapter à celles d'un géosystème à la croissance rapide, semble irrésistible au regard des quelques exemples évoqués ci-dessus; elle est cependant très incomplète et imparfaite et il n'existe pas de cas d'adaptation totale de la maille communale au géosystème urbain actuel, dont les limites sont d'ailleurs très floues. 


\section{Résistances}

Bien que les exemples de modifications du tissu communal au sein des géosystèmes urbains soient très nombreux, les exemples de résistance aux créations en banlieue, comme aux fusions avec la commune centre, le sont encore plus.

Sur la commune d'Avignon, l'écart de Montfavet (fig. 5), comme ses voisins de Morières et du Pontet, a demandé à plusieurs reprises (1829, Entre-deux-guerres et 1989) son érection en commune distincte, se fondant sur "l'inadéquation entre le territoire civique légal où intervient la municipalité et le territoire du quotidien" (Dréano, 1996-1998). Ces tentatives, contrairement à celles de ses voisins, n'ont pas abouti. Ces échecs successifs traduisent d'abord une plus grande proximité de Montfavet par rapport au centre-ville, mais aussi, lors de ces trois tentatives, l'absence d'un réel leader, d'une personnalité forte pour mener les séparatistes.

La création de communes de banlieue lors des premières phases de la croissance urbaine est donc loin d'être systématique, tout comme la fusion de la banlieue avec la commune centre, lors de la croissance urbaine postérieure à la Seconde Guerre mondiale. Le cas parisien, où aucune nouvelle annexion par la commune de Paris n'a été enregistrée depuis 1859 alors que le géosystème urbain parisien s'étend aujourd'hui largement au-delà des limites communales ${ }^{10}$, est à lui seul significatif. Mais il existe aussi de nombreuses grandes villes qui sont de petites communes au sein de vastes agglomérations, faute d'avoir réussi à annexer leurs voisines, au moins les plus proches. Ainsi Nancy, commune de 103605 habitants entourée d'une banlieue de 36 communes regroupant 227758 habitants, ou encore Lille, commune de 184657 habitants dans une banlieue de 62 communes comptant 816243 habitants ${ }^{11}$. Même dans les cas de fusions avérées évoquées dans la partie précédente, l'adaptation de la trame communale au géosystème urbain s'effectue incomplètement. En effet, il n'existe aucun exemple de fusion de toutes les communes périurbaines avec la commune-centre. Les annexions ne concernent donc qu'une partie, souvent faible, des communes de la banlieue, quand ces unions incomplètes ne s'avèrent pas être des échecs. En effet, la commune de Beuvry, qui avait fusionné avec Béthune en 1994, s'en est séparée en 1997. Cet inachèvement de la fusion, et en particulier la résistance de certaines communes, souvent fort peuplées et proches du centre, génère des territoires communaux comportant des enclaves (fig. 6), illustrant l'adaptation incomplète de la trame communale au géosystème. 
Figure 6 : L'adaptation incomplète de la trame communale de l'agglomération dunkerquoise au géosystème urbain (années 1970)

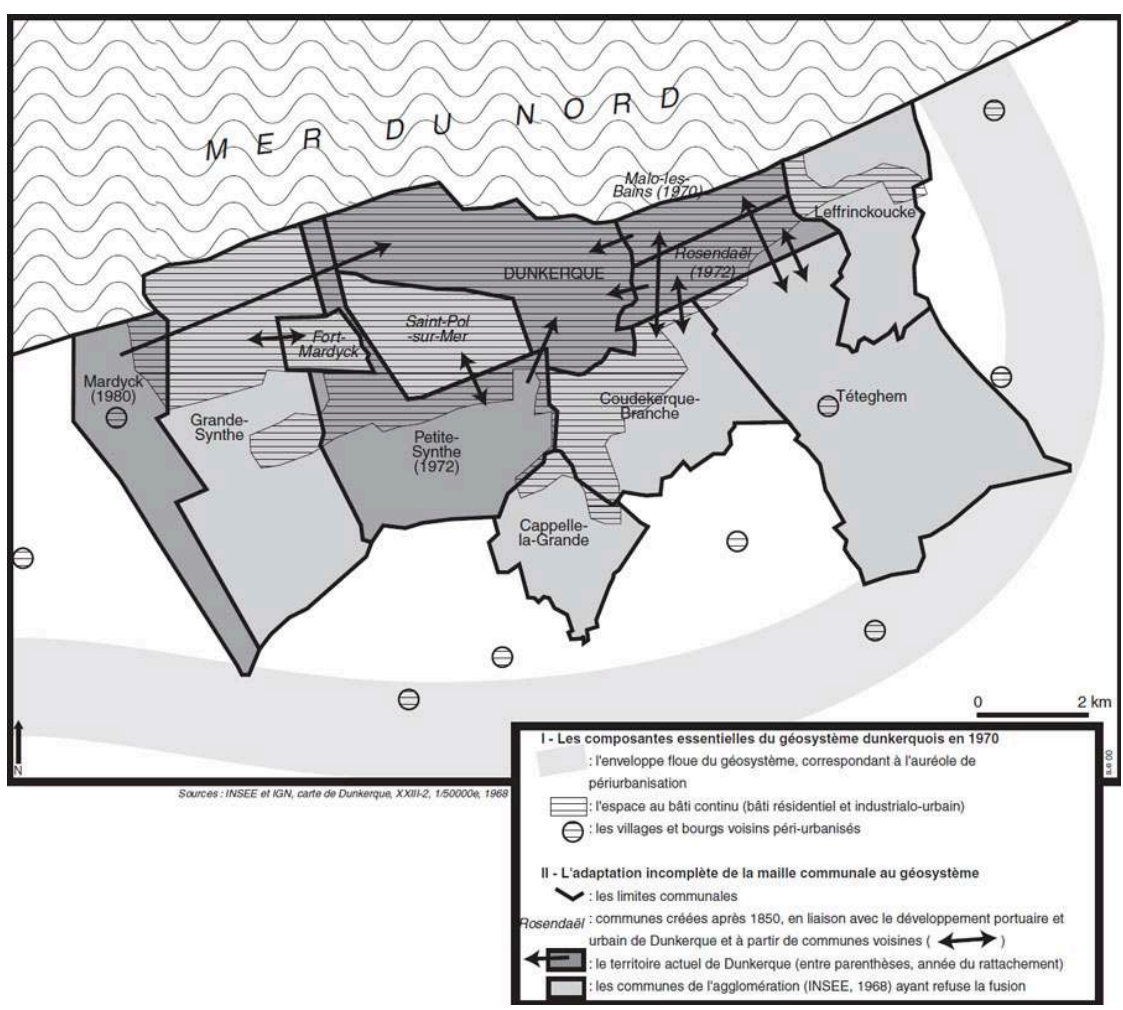

Le mouvement d'adaptation de la maille communale au développement de géosystèmes urbains étendus est donc largement incomplet et s'est en réalité arrêté au début des années 1970, sauf exception. Cependant, l'adaptation de la trame administrative locale au géosystème s'est poursuivie de manière détournée, par la constitution de structures intercommunales qui ne sont pas l'objet de cette étude. Dans le monde rural, l'évolution est évidemment assez différente, mais créations et fusions, liées essentiellement aux mouvements démographiques, cohabitent également.

\section{Le monde rural : flux et reflux}

Au début du XIXe siècle, le monde rural connaissait un maximum démographique et le nombre de communes semblait bien adapté à l'organisation spatiale. Aujourd'hui cependant, c'est en zone rurale que les critiques négatives de la trame communale, émanant souvent des élites urbaines, sont les plus vives. Les nombreuses communes françaises de moins de 100 habitants, voire de moins de 50 habitants paraissent totalement obsolètes aux aménageurs et aux décideurs et ne correspondent évidemment plus à des géosystèmes agricoles depuis longtemps disparus ou anémiés. Pourtant, les tentatives d'adaptation ont été nombreuses.

\section{A. Les créations liées au maximum démographique rural}

On a vu dans la première partie que les ajustements de la trame communale ont été nombreux avant 1815 et que les autorités étaient favorables aux fusions de communes avant 1848. Ce sont néanmoins les créations qui l'emportent, le poids de la 
recomposition territoriale étant plus fort ici que la volonté politique. En Lozère, département pourtant fort dépeuplé aujourd'hui, ce ne sont pas moins de 11 communes nouvelles qui apparaissent entre 1815 et 1914 en milieu rural, contre seulement 2 fusions. Dans l'Aude, ce rapport est de 7 contre 2 et dans l'Isère, de 29 contre 8. L'éloignement géographique et les difficultés de communication entre les écarts et leurs chefs-lieux jouent un rôle dans ce mouvement, mais la naissance de géosystèmes agricoles distincts autour d'écarts grossis par le maximum démographique rural peut également l'expliquer. La croissance démographique leur a finalement permis d'organiser l'espace autour d'elles et de devenir le cœur d'un nouveau géosystème agricole bien distinct du précédent. Ces créations sont toutefois absentes de départements à l'habitat très groupé, sans écart susceptible de grossir et de se détacher de l'organisme d'origine. Dans ce cas, le géosystème agricole déjà en place autour du village chef-lieu, unique agglomération du finage, ne fait que se renforcer. Le maximum démographique rural du milieu du XIXe siècle, combiné à la bienveillance des autorités pour l'émiettement communal perceptible après 1848 , a donc entraîné la naissance de nouvelles communes; le mouvement s'inverse cependant au XXe siècle, notamment après la seconde guerre mondiale.

\section{B. Les fusions et les défusions « Marcellin »}

34 Alors que la fin du XIXe siècle et la première moitié du XXe siècle sont riches en créations communales liées au développement industriel et urbain, les modifications communales sont plus rares sur le front rural et même les communes inhabitées après le premier conflit mondial subsistent longtemps ${ }^{12}$. Ce n'est qu'après la seconde guerre mondiale que se dessine le mouvement de fusion qui connaît son apogée dans la décennie 1970. L'exode rural, entamé depuis près d'un siècle dans certains départements, et la modernisation de l'agriculture, ont en effet vidé de leur substance les géosystèmes agricoles sur lesquels le tissu communal s'était fondé. Le maillage communal du monde rural ne correspond donc plus du tout à l'organisation spatiale, de plus en plus liée à la ville. Une première loi de 1959 simplifie les procédures de rattachement. Puis, la loi Marcellin de 1971, en proposant plusieurs formules de fusions ${ }^{13}$ et surtout des avantages financiers, est censée conduire à la disparition des communes trop petites. Chaque département propose donc un plan de fusion regroupant les communes les plus dépeuplées. Mais, cette fois encore, c'est un échec qui couronne cette tentative puisque seulement $3 \%$ des communes disparaissent. Les résultats sont néanmoins inégaux et certains départements très dépeuplés enregistrent cependant des fusions assez nombreuses. Le record est détenu par la Haute-Marne, avec 110 disparitions entre 1971 et 1974 , soit $20 \%$ du nombre total des communes (fig. 7). 
Figure 7 : Fusions et « défusions » de communes en Haute-Marne après 1959

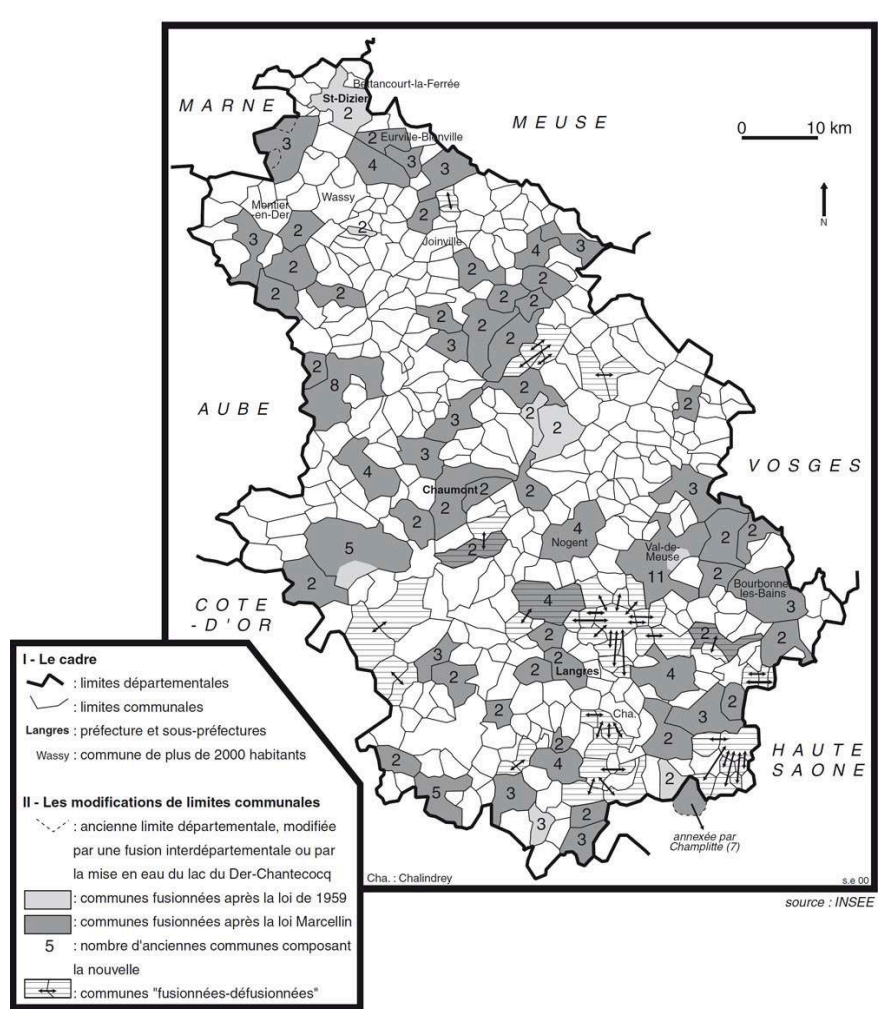

35 Cet échec de la loi Marcellin est en réalité double, car la rareté des fusions est suivie, une dizaine d'années plus tard, par un mouvement de « défusion ». En Haute-Marne, pas moins de 38 communes sont rétablies entre 1979 et 1997, ce qui correspond à un peu plus de $25 \%$ du total des communes disparues suite à la loi Marcellin, peut-être un peu trop favorable à ces divorces (fig. 7). La cause de la séparation n'est pratiquement jamais le développement démographique de la commune rétablie. Dans la Meuse, sur les 20 communes rétablies, seules quatre ont connu, entre leur disparition et leur rétablissement, une croissance de leur population, alors que toutes les autres ont perdu des habitants ; l'une d'elle, Ville-devant-Belrain, passant même de 41 habitants avant la fusion à 18 habitants lors du divorce! Il n'y a donc en aucun cas renaissance d'un géosystème autour de ces communes « défusionnées » et la principale cause, avancée par les différents acteurs, de ces divorces, est le sentiment de perte d'identité de la commune disparue face à la prééminence trop forte du chef-lieu sur sa nouvelle annexe. Cependant, une explication plus globale peut-être avancée. Les plans de fusion, proposés "d'en haut», se sont rarement appuyés sur l'organisation spatiale des communes concernées par ces mouvements. En effet, la plupart de ces petites communes rurales dépeuplées appartiennent aujourd'hui à des géosystèmes plus vastes, dominés par un bourg ou une petite ville proche, le plus souvent un chef-lieu de canton, modeste pôle regroupant les services essentiels et les emplois. Les fusions qui ne se sont pas toujours effectuées autour d'un pôle véritable ont donc le plus souvent échoué, car elles ne correspondaient à aucun territoire économique, social ou culturel. La renaissance de ces petites communes fusionnées-défusionnées s'est en revanche appuyée sur les traces du géosystème agricole lentement mûri depuis des siècles, certes aujourd'hui disparu, mais réelle justification historique et culturelle à l'existence de la commune. Les communes issues de la fusion, avec leurs nouveaux noms ${ }^{14}$ souvent sans 
référence au passé et leur caractère complètement artificiel, n'ont donc souvent pas réussi à effacer cet héritage historique.

Toutes les fusions en milieu rural ne se sont néanmoins pas terminées par un échec; certaines ont réussi, car elles correspondaient mieux à l'organisation spatiale locale, voire à un nouveau géosystème.

\section{De nouveaux géosystèmes, points forts du développement rural}

Les plans de fusions départementaux ont parfois proposé des regroupements communaux autour de chefs-lieux de canton anémiés. Ainsi, en Haute-Marne, 9 communes fusionnent autour de Montigny-le-Roi ${ }^{15}$ entre 1972 et 1974, donnant naissance à la nouvelle commune de Val-de-Meuse (fig. 7). A $25 \mathrm{~km}$ de Langres et $30 \mathrm{~km}$ de Chaumont, Montigny-le-Roi, bourg de quelques centaines d'habitants, polarise incontestablement la vie de Val-de-Meuse par la présence de commerces, services et emplois, créant autour de lui un véritable " pays ", nouveau géosystème, certes dominé par des ensembles urbains plus vastes, comme ceux de Langres ou Chaumont, mais qui possède une réalité spatiale plus évidente que les regroupements purement ruraux et sans véritable pôle attractif. D'autres exemples procèdent du même mouvement, comme le regroupement de 8 communes autour de Vigneulles-lès-Hattonchâtel, petit chef-lieu de canton de la Meuse, ou encore comme l'annexion par Castellanne, dans les Alpes-de-Haute-Provence et en 1973 , de 5 communes voisines très dépeuplées ${ }^{16}$. Là aussi, cette sous-préfecture qui n'atteint pourtant pas 2000 habitants en 1999, est néanmoins, en raison de son grand isolement dans les Préalpes, un pôle local parfaitement capable d'organiser autour de lui un géosystème cohérent.

Certaines fusions autour du chef-lieu de canton ne réussissent pourtant pas. Entre Montigny-le-Roi et Langres, une fusion de 11 communes autour du petit bourg de Neuilly-l'Evêque (Val-de-Gris après la fusion) n'a duré que 12 ans (fig. 7). Dans ce cas, le regroupement communal, peut-être un peu lourd, n'était en réalité pas polarisé par le chef-lieu de canton, mais bien par Langres, distante de moins de $10 \mathrm{~km}$. L'absence d'un géosystème reconnaissable autour de Neuilly-l'Evêque a certainement participé à l'échec de la fusion.

39 Récemment touché par de nombreuses fusions, le monde rural a néanmoins connu quelques créations, anciennes ou récentes, qui ne doivent rien au développement de géosystèmes agricoles. Ces naissances montrent une dernière fois que le tissu communal français n'est pas aussi figé qu'il y parait et que la recomposition territoriale permanente donne naissance à de nouveaux géosystèmes qui s'inscrivent parfois dans la trame administrative par la création de nouvelles communes.

En 1912, des parcelles sont distraites du territoire de Cucq, dans le Pas-de-Calais, pour donner naissance, sur le littoral, à la nouvelle commune du Touquet-Paris-Plage (fig. 8). Cette nouvelle entité administrative, liée à la première vague d'urbanisation touristique sur le littoral de la Manche, à proximité de Paris par le train, est née sur un géosystème touristique saisonnier, émanation estivale des grandes villes émettrices des flux touristiques. Le suffixe «-Paris-Plage » inscrit bien cette nouvelle commune dans la dépendance parisienne, tout comme le rattachement en 1948, toujours au dépens de Cucq, des parcelles sur lesquelles sont bâties l'aérodrome du Touquet, élément constitutif essentiel d'un géosystème touristique. Exception au XIXe siècle, la naissance et le développement de géosystèmes touristiques est plus fréquente au siècle suivant, 
provoquant parfois une modification de la maille communale, tant sur le littoral qu'en montagne. Ainsi, Le Grand-Village-Plage et La Brée-les-Bains sont-elles érigées en communes en 1949 et 1951 sur l'île d'oléron, tout comme Barèges, station pyrénéenne, en 1946, ou enfin Chamrousse, station alpine, en 1989.

Figure 8 : La naissance d'un géosystème touristique : Le Touquet-Paris-Plage, 1912

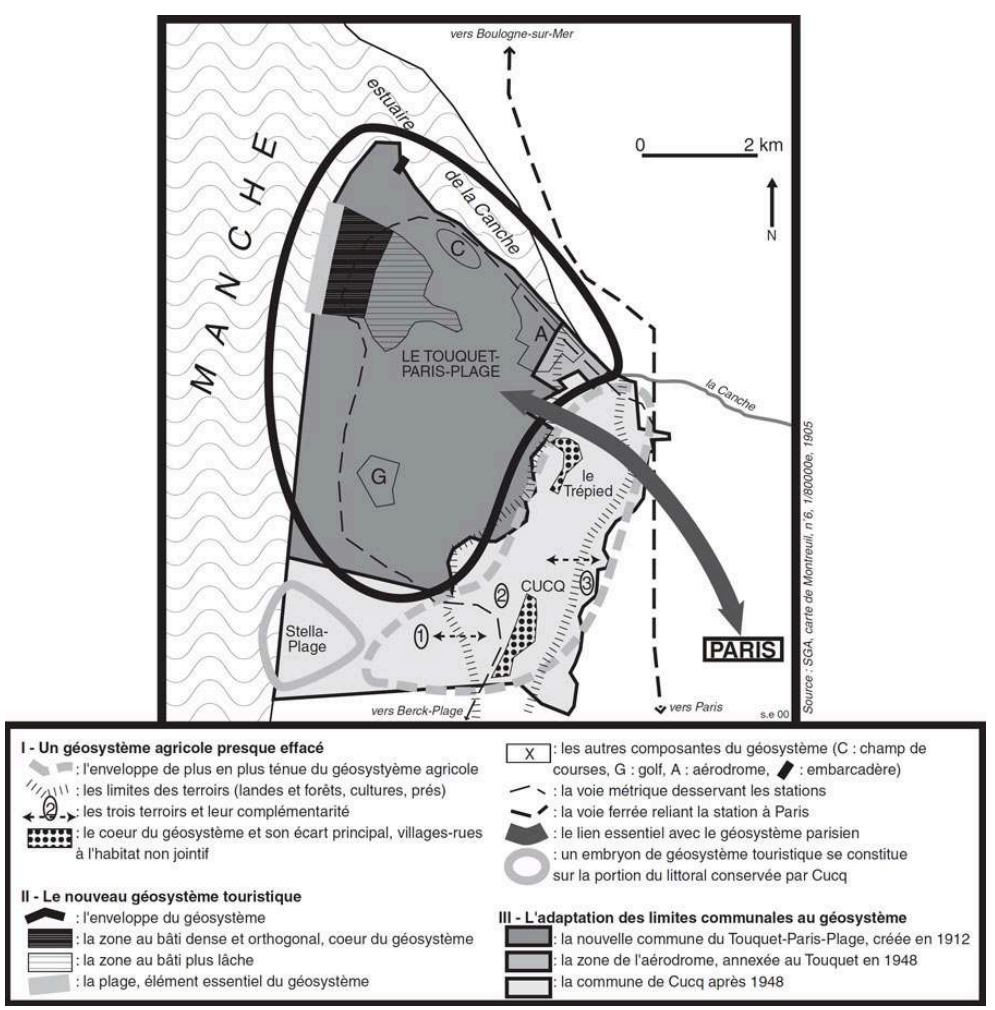

41 Le monde rural a donc été le théâtre de multiples remaniements du tissu communal depuis 1790. Des créations liées au gonflement d'écarts encore agricoles aux fusions ratées des années 1970, réussites et échecs témoignent du tiraillement existant entre une maille administrative à l'évolution lente et une recomposition territoriale permanente, dont les accélérations provoquent parfois une tension telle que les limites communales finissent par s'y adapter.

\section{Conclusion}

L'évolution de l'organisation spatiale, par la naissance ou le déclin de géosystèmes successifs, a donc parfois entraîné une recomposition du territoire politique local. Cette approche devra cependant être renforcée par l'étude ultérieure approfondie d'autres exemples, étayant ou infirmant l'hypothèse de départ.

Bien adaptée à l'organisation spatiale de la fin du XVIIIe et du début du XIXe siècle, la maille communale est aujourd'hui tout à fait inadaptée à l'organisation spatiale urbaine ou rurale actuelle, malgré les ajustements évoqués dans cette étude. L'extraordinaire résistance de ces petites entités parfois très peu peuplées s'explique essentiellement par le fait que la maille communale est devenue en France un véritable patrimoine qui remonte bien au-delà de la Révolution. Les géosystèmes agricoles sur lesquels la grande majorité des communes est née plongent en effet leur racines jusqu'au Moyen-Age, 
voire au-delà, et, aujourd'hui, «ce qui maintient et consolide les communes, ce sont avant tout les valeurs idéologiques et politiques, ainsi que le vécu [...] des individus. Ainsi, avec le temps, le glissement de la détermination majeure qui régit les communes et les villages de France s'est bien effectuée de leur infrastructure économique et matérielle vers leur superstructure idéelle" (Di Méo, 1993). La justification de l'existence des communes n'est donc plus économique ou sociale, mais elle est historique, et la commune est donc devenue, à l'instar d'un monument ou d'un site naturel remarquable, un patrimoine. Cependant, cette inadaptation du territoire politique de la grande échelle à l'organisation spatiale actuelle génère tensions et dysfonctionnements, et même s'il ne semble plus possible aujourd'hui de supprimer d'autorité des milliers de communes pour adapter de force cette maille patrimoniale aux géosystèmes actuels, il reste possible de la contourner par la constitution de structures intercommunales. En effet, «la coopération intercommunale [...] est jusqu'ici la seule expérience menée en Europe d'intégration locale libre sans destruction de l'identité des communes concernées » (Pelatan, 1996). Il faut cependant se garder d'improviser le découpage de ces territoires intercommunaux, en se calquant par exemple sur les limites cantonales, car ces nouveaux territoires, pour fonctionner correctement, doivent eux aussi s'adapter à un géosystème, autour d'un réel pôle rayonnant sur des éléments constitutifs interdépendants.

\section{BIBLIOGRAPHIE}

Beauvalet-Boutouyrie S., Motte C. (1992) - Paroisses et communes de France - Meuse, Dictionnaire d'histoire administrative et démographique, Paris, C.N.R.S. éditions.

Di Meo G. (1991). - La genèse du territoire local : complexité, dialectique et espace-temps, Annales de Géographie, $\mathrm{n}^{\circ}$ 559, Armand Colin, Paris, pp. 273-294.

Di Meo G. (1993). - Les territoires de la localité, l’Espace Géographique, Tome XXII - n4, p. 306-318.

Donze J. (1996). - L'impact des risques technologiques sur l'urbanisation : les communes de Pontde-Claix et de Saint-Fons, Revue de Géographie de Lyon, Vol. 71 - n 1, p. 45-55.

Dreano J.-M. (1996-98). - Affirmation d'une identité locale de banlieue : l'érection en commune du quartier de Montfavet (Avignon), Bulletin de la Société de Géographie de Marseille, Tome XCIII - $\mathrm{n}^{\circ}$ 21, p.69-82.

Dupaquier J., Felkay N., Guerout J., Jacquard J., Lachiver M., Lemee R., Rollet C., Souriac A. (1974). - Paroisses et communes de France - Région parisienne, CNRS éditions, Paris, 922 p.

Edelblutte S. (1997). - Paysages et organisation de l'espace en Lorraine. La vallée de la Moselle d'Épinal à Neuves-Maisons, Thèse, Université de Nancy 2, 495 p.

Edelblutte S. (1998). - Genèse et mutation d'un géosystème industriel, l'exemple de NeuvesMaisons, Côte de Moselle, Revue Géographique de l'Est, Vol. XXXVIII - n 1-2, p. 31-51.

Edelblutte S. (2000). - La Verrerie-de-Portieux, genèse et transformation d'un isolat industrialourbain, Le Pays Lorrain, Vol. 81 - n 4, p. 257-267. 
Edelblutte S. (2001). - Essai sur la genèse du maillage communal en Lorraine (1790-2000), in Territoires, paysages et héritages - Mélanges offerts au professeur Jean-Claude Bonnefont, Université de Nancy2, Centre de Recherches et d'Études sur les Paysages, pp. 125-130.

Ferry C. (1992). - La Blanchisserie Teinturerie de Thaon - 1872-1914, Presses Universitaires de Nancy, Nancy, 304 p.

Humbert A. (1994). - Géographie historique, ou la dérive des systèmes géographiques, Hérodote, n74/75, la Découverte, Paris, p. 95-111.

Libertés, fusions et regroupement de communes (1972), Notes et études documentaires, $\mathrm{n}^{\circ}$ 3899-3900, La documentation française, p. 5-50.

Mathis D. (2001). - Essai sur la genèse du maillage communal en Lorraine (avant 1789), in Territoires, paysages et héritages - Mélanges offerts au professeur Jean-Claude Bonnefont, Université de Nancy 2, Centre de Recherches et d'Études sur les Paysages, p. 116-124.

Pelatan J. (1996). - L'intercommunalité en France, ou l'émergence de nouveaux territoires, in Nouveaux espaces et systèmes urbains, Sedes, Paris, p. 35-42.

Poitou C. (2000). - Paroisses et communes de France - Creuse, CNRS éditions, Paris, 865 p.

Reuter R. (1972), De la ville nouvelle au quartier résidentiel. L'exemple de Saint-Nicolas-en-Forêt, Mosella, Tome $2-\mathrm{n}^{\circ} 4$, p. 30-68.

Reymond H. (1996). - Défense et illustration d'une géographie didactique universitaire. A propos du livre de Jacques Scheibling: Qu'est-ce que la géographie ?, L'Espace Géographique, Tome $25-n^{\circ} 1$, p. 3-23.

De nombreux exemples sont issus de la collection départementale « Paroisses et communes de France - Dictionnaire d'histoire administrative et démographique » (40 volumes parus en 2000) ; seuls les volumes référencés dans le texte sont mentionnés dans la bibliographie.

\section{NOTES}

1. Un rapide dénombrement effectué au début des années 1970 en a relevé près de 12000 (Dupaquier et al., 1974). Encore ne tient-il pas compte des nombreuses fusions-défusions des années 1970-1980...

2. Pour plus de détails sur les modifications de limites communales en Lorraine, voir Edelblutte, 2001.

3. L'exemple bien connu des agglomérations urbaines actuelles, vastes organismes regroupant parfois plusieurs dizaines de communes qui ne s'entendent que difficilement pour la gestion des très nombreux dossiers et problèmes communs, n'a pas besoin d'être ici développé.

4. Dans la Meuse, par exemple, les communes reprennent plus souvent les limites des collectes fiscales que celles des paroisses. En effet, la plupart des annexes de ces paroisses, qui formaient des collectes fiscales différentes de leurs paroisses-mères, sont, comme ces dernières, érigées en communes (Beauvalet-Boutouyrie, Motte, 1992).

5. La comparaison s'effectue entre deux territoires aux limites différentes. Cependant, la France de 1790 était plus petite que celle de l'an 2000, la perte du Nord mosellan étant largement compensée par l'annexion de la Savoie, du Comté de Nice, du Comtat Venaissin et de nombreuses enclaves sur les frontières de l'Est. Enfin, le chiffre de 2000 s'entend sans les communes des départements d'Outre-mer. 
6. De nombreuses fusions ont été proposées sous l'Empire, avec des résultats très inégaux, puisqu'on relève plus de 300 disparitions entre 1811 et 1814 en Moselle, contre 4 seulement dans la Creuse, alors que 141 avaient été proposées...

7. En 1872, dans les Vosges, Thaon, accueillant une vaste usine textile, annexe 25 ha de Girmont, pour que l'usine "n'ait affaire qu'à une seule municipalité et ne soit assujettie qu'à un seul et même régime de contributions et de juridiction " (Ferry, 1992). En échange, Girmont reçoit 8 ha de Thaon...

8. Des communes naissent sur des géosystèmes industriels (le Thillot en 1860 dans les Vosges), mais aussi miniers (Lavaveix-les-Mines dans la Creuse en 1867), voire portuaires (la Nouvelle aujourd'hui Port-la-Nouvelle -, en 1844 dans l'Aude).

9. Un peu moins de 375, relevés entre 1790 et 1974 par Dupaquier et al. (1974).

10. L'agglomération parisienne compte aujourd'hui 395 communes, mais le géosystème s'étend visiblement, entre autres par la rurbanisation, bien au-delà.

11. Compositions des unités urbaines et chiffres issus du recensement de 1999 (INSEE).

12. Certaines de ces communes ( 7 dans la Marne et 2 en Meurthe-et-Moselle) sont rattachées en 1942 à des communes voisines. Seules les communes inhabitées de la Meuse, autour de Verdun, ont été préservées, en raison de la valeur symbolique des combats.

13. Aux côtés de la fusion simple, qui effaçait brutalement la commune absorbée, la «fusionassociation " préserve son identité avec la conservation d'une mairie annexe et d'un maire adjoint et avec la modification du nom de la nouvelle commune issue de la fusion (Notes et études documentaires, 1972).

14. Les fusions ont parfois généré des toponymes aussi insipides que Belles-Forêts (Moselle), Beausite (Meuse) ou Val-de-Gris (Haute-Marne). Les contractions de noms sont aussi assez prisées ; ainsi, dans la Meuse, Ernecourt, Domrémy-aux-Bois et Loxéville sont devenus, après leur fusion, Erneville-aux-Bois.

15. Montigny-le-Roi avait déjà absorbé, en fusion simple, la commune de Meuse en 1966.

16. Castellanne avait déjà annexé en 1948 une commune au chef-lieu noyé sous un lac de barrage (Castillon), ainsi qu'une autre commune très dépeuplée en 1964.

\section{RÉSUMÉS}

Éclairage historique sur la recomposition des territoires, ce travail a pour objectif de montrer que les modifications des limites communales depuis 1790 sont le plus souvent des adaptations du maillage administratif de base à l'évolution de l'organisation spatiale du lieu en question. Une première partie s'attache à présenter et à développer cette idée avant d'évoquer les obstacles s'opposant à cette adaptation. Les parties suivantes, étayées par des exemples précis pris dans toute la France, insistent sur les trois principales causes de modification des limites communales. L'industrialisation, l'urbanisation et le dépeuplement rural ont en effet provoqué des changements mineurs, par simple déplacement de la limite communale, ou majeurs, par la création ou la disparition d'une commune.

Casting an historical light on land reorganisation, this work seeks to demonstrate that modifications to communal boundaries since 1790 are, in most cases, adaptation of the basic administrative system to change in local spacial organisation. This is the idea that will be developed first, before moving on to consider the obstacles that hinder such adaptation. The following sections, illustrated with precise examples from different parts of France, will 
investigate the three main causes of modification to communal boundaries. Industrialisation, urbanisation and rural depopulation have indeed been responsible for minor changes, for example by simple displacement of communal boundaries, or major changes through the creation or disappearance of communes.

Als historischer Rückblick auf die territoriale Neugliederung hat diese Arbeit das Ziel, zu ziegen, daß die Veränderungen der Gemeindegrenzen meistens Anpassungen des administrativen Basisnetzes an die Entwicklung der räumlichen Organisation des jeweiligen Ortes sind. Der erste Teil möchte diesen Gedankengang vorstellen und entwickeln, bevor die Hindernisse aufgezeit werden, die sich dieser Anpassung entgegenstellen. Die folgenden Abschnitte, die sich auf präsize Beispiele aus ganz Frankreich stützen, heben die drei wesentlichen Ursachen der Veränderungen von Gemeindegrenzen hervor. Die Industrialisierung, die Urbanisierung und die Entvölkerung des ländlichen Raumes haben entweder kleinere Veränderungen durch einfache Verlagerung der Gemeindegrenze oder grössere durch Gründung oder Verschwinden einer Gemeinde hervorgerufen.

INDEX

Keywords : commune, geosystem, historical geography, spacial organisation Schlüsselwörter : Gemeinde, Geosystem, historische Geographie, Räumliche Organisation Mots-clés : commune, géographie historique, géosystème, organisation de l'espace

\section{AUTEUR}

\section{SIMON EDELBLUTTE}

Université de Nancy 2 - Centre d'Etudes et de Recherches sur les Paysages (CERPA) - BP 33-97, 54015 Nancy Cedex 